\title{
XVIII.
}

\section{Ueber einige neue Cerverbindungen.}

Von

Dr, L. Th. Lange aus Magdeburg*).

(Bericht aus dem Laboratorium des Dr. Sonnenschein in Berlin.)

Der Umstand, dass die früheren Arbeiten über Cerverbindungen mit einem Gemenge von Cer-, Lanthan- und Didymoxyd angestellt wurden, gab Veranlassung, mit reinem Material einige interessant erscheinende Cerverbindungen darzustellen und ihre Eigenschaften zu studiren.

Bekanntlich ist das Aequivalentgewicht des Cers bestimmt worden von:

$$
\begin{array}{lrr}
\text { Marignac } & \text { zu } & \mathbf{5 9 0 , 8} \\
\text { Beringer } \text { r }^{* * *} \text { ) } & \# & \mathbf{5 7 7 , 0} \\
\text { Hermann } & \mathbf{5 7 * *} \text { ) } & \mathbf{5 7 5 , 0} \\
\text { Rammelsberg } & , & \mathbf{5 7 2 , 8} \\
\text { Otto }+\dagger) & " \mathbf{5 7 8 , 8} \\
\text { Jegel }+\dagger \dagger & , & \mathbf{5 7 5 , 8}
\end{array}
$$

Als Durchschnitt wurde bei der Berechnung der in dieser Arbeit vorkommenden Zahlen das Aequivalentgewicht des Cerium zu 575, wenn $O=100$ und 46 wenn $\mathrm{H}=\mathbf{1}$ ist, angenommen.

Das zur Darstellung der in dieser Arbeit beschriebenen Verbindungen und Salze angewandte Cer war nach der von $\mathrm{Hermann}$ und Marignac zuerst angewandten und

*) Durch Zufall verspätct.

**) Ann, d. Chem, u. Pharm. LXVIII, 212.

***) Ebendas. XLII, 140.

$\left.{ }^{* * * *}\right)$ Dies. Journ. XXX, 186.

f) Pogg. Ann. LXV, 66.

tf) Ebendas. XL, 404.

tit) Dies. Journ. LXXIII, 200.

Jouru. 1. prakt. Chemie. LXXXII. 3. 
von Holtzmann*) nach der Bunsen'schen**) Methode vervollständigten Trennungsart abgeschieden.

Dieselbe soll hier nur kurz angeführt werden, um die Mittheilung einiger dabei gemachter Beobachtungen daran zu knüpfen. Als Rohmaterial diente schwedischer Cerit.

Der fein gepulverte Cerit wurde mit concentrirter Schwefelsäure zu einer breiartigen Masse angerührt und erhitzt. Die stark aufgeblähte und völlig trocken gewordene Masse wurde zerstossen und nach und nach unter Unrühren, um Erhitzung zu vermeiden, in kaltes Wasser eingetragen. Hierbei wurde eine ziemlich bedeutende Wasserstoffgasentwickelung beobachtet, das Gas stieg besonders beim Umrühren der Masse selbst nach Tage langem Stehen in so grossen Blasen auf, dașs dieselben aufgefangen und angezündet werden konnten; sie verbrannten unter Knall mit schwach gelblicher Flamme. Als nach dreivierteljährigem Liegen des so ausgezogenen Cerits derselbe wiederum mit concentrirter Schwefelsäure zur Trockne eingedampft wurde, wurde auch hier beim Uebergiessen mit Wasser eine deutliche Wasserstoffentwickelung bemerkt, während noch viel Ceroxyd gelöst wurde. Der kalte Auszug wurde bis zum Sieden erhitzt und das sich hierbei krystallinisch ausscheidende Salz aus der heissen Flüssigkeit gezogen, mit siedendem Wasser abgewaschen und dann in kaltem Wasser gelöst. Aus diesser Flüssigkeit wurden durch Schwefelwasserstoff die Spuren von Kupfer, Molybdän und Wismuth gefällt, welche den Krystallen anhaften blieben. Nach Abfiltrirung der Schwefelmetalle wurde nach Zusatz von Salzsäure und nach Hindurchleiten von Chlor, um das durch Schwefelwasserstoff reducirte Eisenoxydul wieder zu oxydiren, die Ceritoxyde durch Oxalsäure gefällt. Die hierbei erhaltenen Mutterlaugen wurden nicht zu den in dieser Arbeit beschriebenen Salzen verwandt.

Die auf diese Weise gereinigten Ceritoxyde wurden noch decantirt und auf dem Filter mit heissem Wasser

*) Dies. Journ. LXXIII, 200.

**) Dies, Journ. LXXV, 321. 
ausgewaschen, alsdann mit dem halben Gewicht reiner Magnesia alba zu einem Brei angerührt, getrocknet und in einer grossen Platinschale so lange dem Gebläsefeuer unter Umrühren ausgesetzt, bis die ganze Masse glühte. Man muss sich hüten, auch nur eine kleine Spur von Oxalsäure unzersetzt zu lassen, weil sonst beim Auflösen in Salpetersäure das Ceroxydoxydul reducirt wird und sich nicht abscheiden lässt. Die geglühten Oxyde wurden in concentrirter siedender Salpetersäure gelöst, was sehr viel Schwierigkeit hat, da das geglühte Ceroxyd sich nur in ganz concentrirter Salpetersäure löst und die Lösung doch nicht $\mathrm{zu}$ sehr concentrirt werden darf, wenn man Krystalle erhalten will, weil sonst die ganze Masse beim Erkalten fest wird; auch darf man kein Wasser hinzusetzen, weil sich sonst unlösliches basisches Salz abscheidet.

Hierbei wurde die dunkelgelbrothe Flüssigkeit auf einem solchen Concentrationsgrad erhalten, dass sich nach 24 stündigem Stehen Krystallmassen bildeten. Manche von den erhaltenen Krystallen hatten einen Durchmesser von $\frac{1}{2}-\frac{3}{4}$ Zoll. Diese Krystalle wurden zu je $100 \mathrm{Grm}$. in 100 C.C. kalten Wassers gelöst. Sollte sich hierbei Trübung zeigen, so muss man das Klare durch Stehenlassen und $\mathrm{Abgiessen}$ zu gewinnen suchen, da beim Filtrìren das Ceroxydoxydul leicht durch das Papier zu Oxydul reducirt wird und als solches sich alsdann nicht abscheiden lässt.

Diese Lösung wurde in 2 Liter mit 12 C.C. Schwefelsäure angesäuertes siedendes Wasser eingetragen, wobei sich basisch schwefelsaures Ceroxyd abschied, das durch Decantiren mit siedendem schwefelsäurehaltigen Wasser mehrere Male gereinigt wurde.

Das auf diese Weise von Didym und Lanthan völlig befreite schwefelsaure Ceroxydoxydul wurde durch Kochen mit Aetzkali in Ceroxyduloxydhydrat übergeführt und durch längeres anhaltendes Decantiren mit siedendem. Wasser völlig von Kali und Schwefelsäure befreit.

Der Anfangs röthliche Niederschlag fing hierbei von oben an schwefelgelb zu werden und beim Trocknen verwandelte sich die ganze Masse in ein staubiges Pulver von schwefelgelber Farbe. 
Als von diesem gelben Pulver eine Quantität 4 Stunden bei $150^{\circ}$ getrocknet wurde und hicrvon $4,339 \mathrm{Grm}$. geglüht wurden, so ergab sich beim nachherigen Wägen ein Verlust von 0,267 Grm. $=6,15$ p.C. Das Fehlende war zum grossen Theil Kohlensäure, denn das nur getrocknete Ceroxydoxydul brauste beim Uebergiessen mit Schwefelsäure stark auf, das geglühte nicht mehr. Auffallend war es, dass das so erhaltene gelbe Ceroxydoxydul beim Digeriren sowohl mit Schwefelsäure als auch mit Salpetersüure einen chlorähnlichen Geruch entwickelte, obgleich in der ganzen zweiten IIälfte der Bearbeitung keine Salzsäure angewendet war. Das gelbe Pulver wurde beim starken Glühen röthlich ebenso wie der Niederschlag von Kali in Ceroxyduloxydlösungen gleich nach der Fällung.

Interessant ist es, dass dieses so bereitete Ceroxyduloxyd, getrocknet und gepulvert in einen Strom von Schwefelwasserstoffgas geschüttet, dieses entzündet, indem es sich in Oxydul und zu Cersulfuret umwandelt.

Diese Reaction geht schon vor sich in der Kälte und in feuchtem Schwefelwasserstoffgas.

Leitet man in einer Glasröhre trockenes Schwefelwasserstoffsas über getrocknetes Ceroxydoxydul, so wird das schwefelgelbe Ceroxyduloxyd unter Erhitzen in ein schmutzig grünlich graues Pulver verwandelt. Am andern Ende der Röhre ist nicht eher eine Spur von Schwefelwasserstoff wahrzunehmen, bis särmmtliches Ceroxyduloxyd zersetzt ist, mag der hindurchgehende Strom von Schwefelwasserstoffgas auch noch so stark sein.

Unterbricht man nach vollständiger Zersetzung den Strom von Schwefelwasserstoff und leitet ebenfalls getrocknetes Wasserstoffgas durch die Röhre und erwärmt diese, so werden Wasser- und Schwefeldämpfe entwickelt.

Bei einem derartigen Versuche wurden in einer Kugelröhre 4,031 Grn. geglühten Ceroxyduloxyds in einem Strome trockenen Schwefelwasserstoffs bis zum schwachen Rothglühen erhitzt. Nachdem alles Wasser und aller Schwefel aus der Röhre verjagt war, wurde dieselbe wieder gewogen und ergab eine Gewichtszunahme von 0,049, woraus sich der Gehalt an Cersulfuret gleich 45 p.C. ergiebt. 
Bei einem anderen vollständig gleich angestellten Versuche vermehrten sich $3,165^{\circ}$ Grn. geglühten Ceróxyduloxyds um 0,008 , was auf einen Gehalt von 18,84 p.C. Cersulfuret in dem erhaltenen Producte schliessen lässt.

Bei vierfach wiederholtem Versuche war es nicht möglich, ein constantes Resultat zu erhalten, obgleich die Neigung, sich mit Schwefelwasserstoff zu zersetzen, selbst dem sonst so schwer angreifbaren geglühten Ceroxyduloxyd eigen ist.

Wird das in der Kälte durch Schwefelwasserstoff erhaltene Gemenge von Ceroxydul und Cersulfuret mit Sauerstoff in Berührung gebracht, so oxydirt sich dasselbe zu Ceroxyduloxyd, und zwar im feinvertheilten Zustande unter Feuererscheinung, in dichteren Massen ohne eine solche, jedoch immer unter starkem Erhitzen und Entwickelung von schwefliger Säure, ja sogar im feuchten Zustande und unter Wasser.

Wird Ceroxyduloxyd in Schwefelsäure gelöst, so entsteht eine rothgelbe Flüssigkeit, welche äusserst oxydirende Eigenschaften besitzt, da dieselbo selbst in den verdünntesten Lösungen Eisenoxydul augenblicklich in Eisenoxyd, Kaliumeisencyanür in Kaliumeisencyanid umwandelt, und aus Jodkalium das Jod frei macht, so dass es als Oxydationsmittel in der Maassanalyse Anwendung finden kann.

Da das Manganoxydul erst nach längerer Zeit durch dasselbe oxydirt wird, so dürfte es sich zum Titriren des Eisens besonders in dem Falle eignen, wo kein Manganoxydul zugegen ist, da die Gegenwart aller anderen Metalle durchaus nicht schadet, und nach vollendeter Oxydation die intensive Farbe des schwefelsauren Ceroxyduloxyds hervortritt. Was die Beständigkeit des Titers betrifft, so scheint diese Lösung das übermangansaure Kali bei weitem $\mathrm{zu}$ übertreffen, da das schwefelsaure Ceroxydul bei Anwesenheit von desoxydirenden Körpern durchaus keine Neigung zeigt, sich zu zersetzen. Es muss nur die Bildung eines basischen Salzes vermieden werden, was durch einen passenden Säurczusatz geschehen kann, 
da ein Ueberschuss von Säure ohne Einfluss auf die Reaction ist. $\Lambda \mathrm{m}$ besten wendet man Schwefelsäure an.

Das zum Titriren benutzte Ceroxyd kann durch Fällen mit Oxalsäure u. s. w. wieder gewonnen werden. Der Titer wird auf gewöhnliche Weise durch Eisendraht oder durch schwefelsaures Eisenoxydulammoniak bestimmt.

\section{Verbindungen des Cers mit den Salzbildern.}

I. Cerjodür. Getrocknetes Ceroxyduloxyd löst sich in Jodwasserstoffsäure mit Leichtigkeit unter Abscheidung von Jod auf. Verwandelt man das freie Jod durch Schwefelwasserstoff in Jodwasserstoffsäure und dampft die Flüssigkeit mit überschüssigem Ceroxyduloxyd unter fortwährendem Durchleiten von Schwefelwasserstoff ein, so erhält man nach dem Filtriren eine völlig farblose Lösung von Cerjodür, die sich an der Luft bräunt, über Schwefelsäure aber an der Oberfläche dünne, farblose und wasserhelle Krystalle absetzt, die an der Luft zu einer braunen Lösung zerfliessen.

Zur Untersuchung wurden $0,787 \mathrm{Grm}$. in Wasser gelöst, mit Salpetersäure angesäuert und das Jod mit salpetersaurem Silberoxyd als Jodsilber gefällt. Das Jodsilber auf einem gewogenen Filter abfiltrirt und bei $120^{\circ}$ getrocknet ergab $0,806 \mathrm{Grm}$. Ag.J $=0,43544 \mathrm{Grm}$. J.

Nachdem das überschüssig zugesetzte Silber durch Salzsüure gefällt und diess abfiltrirt war, wurde das Cer durch oxalsaures Ammoniak gefällt; abfiltrirt und geglüht ergab es $0,202 \mathrm{Grm}$. $\mathrm{CeO}+\mathrm{Ce}_{2} \mathrm{O}_{3}$ entsprechend $0,1642 \mathrm{Ce}$.

Hieraus berechnet sich die Formel CeJ $+6 H O$.

$\begin{array}{lcc} & \text { Berechnet. } & \text { Gefunden. } \\ \text { Jod } & \mathbf{5 6 , 9 2} & \mathbf{5 5 , 3 3} \\ \text { Cerium } & \mathbf{2 0 , 5 3} & \mathbf{2 0 , 8 6} \\ \text { 6HO } & \mathbf{2 3 , 8 1} & -\end{array}$

Als gleiche Theile von Jodwasserstoffsäure, der eine mit Ammoniak, der andere mit Ceroxyduloxyd unter Hindurchleiten von Schwefelwasserstoff neutralisirt wurden, dann beide Theile gemengt, eingedampft und filtrirt, krystallisirten über Schwefelsäure kleine glänzende Würfel aus der Flüssigkeit, wolche sich längere Zeit an der Luft 
hielten und in einem einfach mit Korkstöpseln verschlossenen Glasrohre selbst nach einem Vierteljahr ihren Glanz und ihre Festigkeit behielten, jedoch nach und nach etwas braun wurden. Bei der Analyse ergab das Salz Jodammonium mit nur $\frac{1}{2}$ p.C. Cerium.

II. Cerchlorür. Als ein Gemenge von Chlorwasserstoffsäure und Eisenblausäure mit Ceroxyduloxyd im Ueberschuss digerirt wurde, entstand ein Cerchlorür, welches ganz andere Eigenschaften zeigte, als das aus Ceroxyduloxyd und Salzsäure dargestellte. Es war nämlich die so gewonnene Verbindung völlig farblos und ron ganz anderer Krystallform als die auf die gewöhnliche Weise dargestellte.

Zur Untersuchung dieses Körpers wurden 1,247 Grm. in Wasser gelöst, mit Salpetersäure angesäuert, mittelst salpetersaurem Silberoxyd das Chlor gefällt; das bei $120^{\circ}$ getrocknete Chlorsilber wog 1,452 Grm. entsprechend 0,359 Grm. Chlor. In dem Filtrat wurde mit Salzsäure das überschüssige salpetersaure Silberoxyd gefällt und abfiltrirt, dann das Cer durch oxalsaures Ammoniak gefüllt, abfiltrirt geglüht gab sie $\mathbf{0 , 5 7 3 4}$ Ceroxyduloxyd $=0,466 \mathrm{Ce}$ rium. Hieraus berechnet sich die Formel:

\begin{tabular}{llc}
\multicolumn{3}{c}{ 2CeCl $+\mathbf{9 H O}}$. \\
Berechnet. & Gefunden. \\
Cerium & $\mathbf{3 7 , 7 0}$ & $\mathbf{3 7 , 3 7}$ \\
Chlor & $\mathbf{2 9 , 0 1}$ & $\mathbf{2 8 , 8}$ \\
Wasser & $\mathbf{3 3 , 2}$ & -
\end{tabular}

Verbindungen des Cers mit Cyan.

Schwefelsaures Ceroxydul giebt mit Kaliumeisencyanür einen weissen, an der Luft'bläulich werdenden Niederschlag. Beim Trocknen wird dieser Niederschlag pyrophorisch und verglimmt bei hinreichendem Luftzutritt schnell durch die ganze Masse unter Zurücklassung von Eisenoxyd und Ceroxyduloxyd.

Selbst als ein Theil Ceriumeisencyanür mit $\mathbf{3}$ Theilen Kaliumeisencyanür innig gemengt wurde, fing doch das Gemenge beim Trocknen an zu verglimmen. Als eben solches Gemenge in einem Porcellantiegel fest eingestampft 
und bedeckt geglüht wurde, zog Alkohol nach dem Erkalten nur Cyankalium aus, der Rückstand war ein schmutzig graues Pulver, ein inniges Gemenge (oder Verbindung?) von Ceroxydul mit Eisen.

Durch directe Einwirkung von Ferrocyanwasserstoffsäure auf Ceroxydul wurde kein Ceriumeisencyanür erhalten.

\section{Salpetersaures Ceroxydul.}

Löst man Ceroxyduloxyd unter Anwendung reducirender Substanzen, z. B. Alkohol, in concentrirter Salpetersäure, so erhält man nach Abfiltrirung des ungelösten Ceroxyduloxyds eine farblose Lösung, welche eingedampft zu einer klaren, farblosen, syrupartigen Masse wird, die beim Erkalten krystallinisch erstarrt. Es hat eine schwache rosa Farbe und zieht leicht Feuchtigkeit an.

Diese krystallinische Masse längere Zeit über Schwefelsäure und Chlorcalcium stehen gelassen enthält 4 Aeq. Wasser. 1,096 Grm. hiervon aufgelöst und mit oxalsaurem Ammoniak das Ceroxydul darin gefällt, dieses abfiltrirt und geglüht ergab $0,447 \mathrm{Grm}$. Ceroxyduloxyd $=\mathbf{0 , 4 2 4 7}$ Ceroxydul. Diess entspricht 38,75 p.C. Ceroxydul, woraus sich die Formel $\mathrm{CeONO}_{5}+4 \mathrm{HO}$ ergiebt.

$\begin{array}{lcc} & \text { Berechnet. } & \text { Gefunden. } \\ \text { Ceroxydul } & \mathbf{3 7 , 5} & \mathbf{3 8 , 7 5} \\ \text { Salpetersäure } & \mathbf{3 7 , 5} & - \\ \text { Wasser } & \mathbf{2 5} & -\end{array}$

Bei $150^{\circ}$ C. 3 Stunden lang getrocknet, verliert es ungefähr 2 Aeq. HO. Bei $200^{\circ}$ wird es zersetzt.

\section{Salpetersaures' Ceroxydul-Kali.}

Vermischt man eine Lösung von salpetersaurem Ceroxydul mit salpetersaurem Kali und dampft diese ein, so lässt sie sich sehr stark concentriren, ehe sich Salz abscheidet. Aus der syrupdicken Lösung krystallisiren über Schwefelsäure kleine glänzende Krystalle des Doppelsalzes, die aber nicht jedesmal gleich zusammengesetzt sind, da bei mehreren Analysen ganz verschiedene Resultate gefunden wurden. Eine Lösung des Doppelsalzes, aus der 
viel Salpeter auskrystallisirt war, wurde von diesem abgegossen und über Schwefelsäure vierzehn Tage hingestellt. Die gebildeten völlig farblosen Krystalle zwischen Fliesspapier gepresst und über Schwefelsäure getrocknet wurden untersucht.

1,546 Grm. davon bei $180^{\circ}$, einer Temperatur, bei welcher die wenigsten Ceroxydulsalze zersetzt werden, 6 Stunden lang getrocknet ergaben einen Verlust von 0,114 Grm., der als Wasser berechnet wurde. Der Rückstand wurde längere Zeit geglüht, dann in Wasser gelöst und das ungelöste Ceroxyduloxyd abfiltrirt wog 0,473 gleich 0,4512 Ceroxydul. Das Filtrat wurde zur Trockne ververdampft, mit Platinchlorid versetzt, im Wasse bade zur Trockenheit gebracht und mit Alkohol ausgezogen auf einem getrockneten und gewogenen Filter abfiltrirt und bei $110^{\circ} 4$ Stunden getrocknet ergab 1,321 Grm. Kaliumplatinchlorid entsprechend 0,255138 Grm. Kali. Hieraus folgt die Formel:

\begin{tabular}{lcc}
\multicolumn{2}{c}{$\mathrm{KONO}_{5}+3 \mathrm{CeONO}_{5}+4 \mathrm{HO}}$. \\
& Berechnet. & Gefunden \\
Kali & $\mathbf{1 6 , 7 2}$ & $\mathbf{1 6 , 5 0}$ \\
Ceroxydul & $\mathbf{2 8 , 8 2}$ & $\mathbf{2 9 , 1 8}$ \\
Wasser & $\mathbf{6 , 4 0}$ & $\mathbf{7 , 3 7}$ \\
$\mathrm{NO}_{5}$ & $\mathbf{4 8 , 0 4}$ & -
\end{tabular}

\section{Salpetersaure Ceroxydul-Magnesia. \\ $\mathrm{CeONO}_{5}+\mathrm{MgONO}_{5}+8 \mathrm{HO}$.}

Dieses Salz wird erhalten durch Auflösen gleicher Aequivalente Ceroxyduloxyd und Magnesia in Salpetersäure unter Anwendung reducirender Substanzen, z. B. Alkohol. Es ist schwach rosa gefärbt, in Wasser und Alkohol sehr leicht löslich und kann in grossen Krystallen erhalten werden. Bei $110^{\circ}$ verliert es einen Theil seines Krystallwassers, den Rest erst bei $200^{\circ}$, wobei es zu einer glasigen Masse schmilzt, alsdann nach dem Erkalten mit Wasser befeuchtet, zieht es dasselbe begierig unter Erwärmung an, ohne sich jedoch klar zu lösen.

Zur Analyse wurden 1,128 $\mathrm{Grm}$. angewandt; darin das Ceroxydul mit Oxalsäure gefilllt, abfiltrirt und geglüht er- 
gab 0,247 Ceroxyduloxyd $=\mathbf{0 , 2 3 5 6}$ CeO. In dem Filtrat wurde mit phosphorsaurem Natron und Ammoniak die Magnesia ais phosphorsaure Ammoniak-Magnesia gefällt; abfiltrirt und geglüht gab sie 0,2422 $\mathrm{MgOPO}_{5}=0,0872 \mathrm{MgO}$. Das Wasser wurde in einer besonderen Quantität bestimmt. $0,670 \mathrm{Grm}$. wurden zuerst 2 Stunden bei $110^{\circ}$ getrocknet; diess ergab einen Gewichtsverlust von $\mathbf{0 , 0 7 1}$, alsdann noch 3 Stunden bei $200^{\circ}$ getrocknet stieg der Gewichtsverlust auf 0,206. Hieraus ergiebt sich die Formel:

$$
\mathrm{MgONO}_{5}+\mathrm{CeONO}_{5}+8 \text { aq. }
$$

$\begin{array}{lcc} & \text { Berechnet. } & \text { Gefunden. } \\ \text { Ceroxydul } & \mathbf{2 1 , 2 6} & \mathbf{2 0 , 8 8} \\ \text { Magnesia } & \mathbf{7 , 8} & \mathbf{7 , 7 3} \\ \text { Salpetersäure } & \mathbf{4 2 , 6} & - \\ \text { Wasser } & \mathbf{2 8 , 3 4} & \mathbf{2 7 , 7 6}\end{array}$

Nach 2 stündigem Trocknen bei $110^{\circ}$ waren genau 3 Aeq. HO verloren. In Procenten:

$\begin{array}{cc}\text { Berechnet. Gefunden. } \\ \mathbf{1 0 , 6 3} & \mathbf{1 0 , 6}\end{array}$

\section{Salpetersaures Ceroxydul-Manganoxydul.}

Löst man kohlensaures Manganoxydul in Salpetersäure und fügt $\mathrm{zu}$ der Lösung salpetersaures Ceroxydul und Alkohol, so krystallisirt nach dem Eindampfen das Doppelsalz beim Erkalten in prächtigen Krystallen von zart rosenrother Farbe und von einer Grösse bis zu einem Zoll im Durchmesser aus der Flüssigkeit. Die Krystallc wurden in Wasser gelöst, die Flüssigkeit eingedampft und unter Umrühren erkalten gelassen, so dass sich nur kleine Krystalle bilden konnten; diese wurden zwischen Fliesspapier gepresst und über Schwefelsäure getrocknet.

Zur Analyse wurden 1,818 Grm. dieser kleinen Krystalle verwandt; diese gelöst, mit Salpetersäure angesäuert, mit Oxalsäure das Ceroxydul gefällt, dieses nach 24 stündigem Stehen abfiltrirt und geglüht ergab $0,376 \mathrm{Grm}$. Ceroxyduloxyd, entsprechend 0,3587 Ceroxydul. Das Filtrat mit kohlensaurem Natron neutralisirt und das Manganoxydul eben damit gefällt, abfiltrirt, ausgewaschen und geglüht ergab 0,273 Grm. Manganoxyduloxyd, gleich 
0,2539 Grm. Manganoxydul. Das Wasser wurde in einer besonderen Quantität bestimmt.

1,402 Grm. des Doppelsalzes bei $150^{\circ} 4$ Stunden lang getrocknet ergab einen Gewichtsverlust von $0,178 \mathrm{Grm}$. Hieraus folgt die Formel:

\begin{tabular}{lcc}
\multicolumn{3}{c}{$\mathrm{CeONO}_{5}+\mathrm{MnONO}_{5}+8 \mathrm{HO}}$. \\
& Berechnet. & Gefunden. \\
Ceroxydul & $\mathbf{2 0}$ & $\mathbf{1 9 , 7 3}$ \\
Manganoxydul & $\mathbf{1 3 , 1 4}$ & $\mathbf{1 3 , 9 7}$ \\
Salpetersäure & $\mathbf{4 0}$ & - \\
Wasser & $\mathbf{2 6 , 6 6}$ & $\mathbf{1 2 , 6 8}$
\end{tabular}

Das Salz verliert also bei $150^{\circ}$ nur 4 Aeq. HO, doch tritt schon hierbei Zersetzung ein, indem sich das Salz schwärzt von ausgeschiedenem Manganoxyd durch die Einwirkung der Salpetersäure auf das Manganoxydul.

\section{Salpetersaures Ceroxydul-Kobaltoxydul.}

Eine Lösung von salpetersaurem Ceroxydul mit salpetersaurem Kobaltoxydul vermischt, lässt sich sehr stark concentriren, ehe sie beim Erkalten Krystalle fallen lässt. Erst nach längerem Stehen über Schwefelsäure und Aetzkali konnten dieselben erhalten werden. Die grösseren Krystalle des Doppelsalzes sind braun, die kleineren rubinroth, an der Luft zerfliessen sie und verwittern über Schwefelsäure.

Zur Untersuchung dieses Salzes wurden 0,415 Grm. in Wasser gelöst, die Lösung stark verdünnt und mit sehr viel Salmiak versetzt, hlerauf mit Ammoniak das Ceroxydul gefällt, dieses wiederum in Salzsäure gelöst, verdünnt, mit viel Salmiak versetzt und wieder mit Ammoniak gefallt, abfiltrirt, geglüht, und ergab $0,0915 \mathrm{Grm} . \mathrm{C}_{3} \mathrm{O}_{4}$, entsprechend $0,08586 \mathrm{CeO}=20,68$ p.C. Cerosydul. Die von den Niederschlägen abfiltrirte Flüssigkeit wurde mit Schwefelammonium gefällt, das Schwefelkobalt abfiltrirt, in Salpetersäure gelöst und zur Trockne eingedampft, wiederholentlich mit Salpetersäure und Schwefelsäure befeuchtet und eingedampft, schliesslich schwach geglüht, bis zur vollständigen Verflüchtigung der überschüssigen Schwefelsäure, gewogen ergab $0,114 \mathrm{CoOSO}_{3}$, entsprechend 0,05516 
$\mathrm{CoO}=13,29$ p.C. Kobaltoxydul. Die Salpetersäure wurde mittelst gewogenen Kupferblechs und überschüssiger Salzsäure in einem Strome Kohlensäuregases bestimmt mit der Vorsicht, dass die in dem ersten Kolben auftretenden Dämpfe in einen zweiten traten, worin ebenfalls Salzsäure und ein gewogenes Kupferblech sich befanden, welches auch hier merklich angegriffen wurde, als Zeichen, dass unzersetzte Salpetersäure oder Chlor aus dem ersien Kolben, in welchem sich die Substanz befand, mit übergegangen waren. Bei $0,2285 \mathrm{Grm}$. Substanz waren $0,303 \mathrm{Grm}$. Kupfer $\mathrm{zu}$ Chlorür gelöst, was $\mathbf{0 , 0 8 6 1 7 3 2} \mathrm{Grm}$. $\mathrm{NO}_{5}$ entspricht $=$ 37,71 p.C. Salpetersäure, während die Rechnung 39,78 p.C. verlangt. Hieraus ergiebt sich die Formel:

\begin{tabular}{lcc}
\multicolumn{3}{c}{$\mathrm{CeONO}_{5}+\mathrm{CoONO}_{5}+\mathbf{8 H O}}$. \\
Berechnet. & Gefunden. \\
$\mathrm{CeO}$ & $\mathbf{1 9 , 8 9}$ & $\mathbf{2 0 , 6 8}$ \\
$\mathrm{CoO}$ & $\mathbf{1 3 , 8 1}$ & $\mathbf{1 3 , 2 9}$ \\
$2 \mathrm{NO}_{5}$ & $\mathbf{3 9 , 7 8}$ & $(\mathbf{3 7 , 7 1})$ \\
$8 \mathrm{HO}$ & $\mathbf{2 6 , 5 1}$ &
\end{tabular}

\section{Salpetersaures Ceroxydul-Nickeloxydul.}

Eine auf gleiche Weise wie die vorigen Salze dargestellte Lösung von salpetersaurem Ceroxydul mit salpetersaurem Nickeloxydul krystallisirte nach dem Erkalten in schön smaragdgrünen Krystallen bis zu Zollgrösse, die sich sowohl an der Luft als auch über Schwefelsäure sehr gut erhalten.

Zur Analyse wurden $\mathbf{0}, 921 \mathrm{Grm}$. des Doppelsalzes ancr. wandt, dieses in Wasser gelöst, mit Ammoniak gefillt, abfiltrirt, ergab 0,198 $\mathrm{Ce}_{3} \mathrm{O}_{4}$, entsprechend 0,1959 $\mathrm{CeO} \stackrel{ \pm}{=}$ 21,06 p.C. Ceroxydul. Als dasselbe wieder in Schwefel. säure aufgelöst wurde und mit Ammoniak gefällt, wurde das Filtrat durch Schwefelammonium nicht dunkler gefärbt.

Da die directe Bestimmung des Nickeloxyduls verunglückte, so wurden $0,54 \mathrm{Grm}$. des Doppelsalzes vorsichtig erhitzt bis zum Glühen; der Rückstand wog 0,1895=35,09 p.C. Verändert man hierin das eine $\Lambda$ equivalent zu Ceroxydul, so bleibt für. Nickeloxydul und Ceroxydul 34,08; 
zieht man hiervon das gefundene Ceroxydul $a b$, so bleibt für Nickeloxydul 13,02 p.C. Die Salpetersäurebestimmung wurde, wie beim Zinksalze ausführlich beschrieben wird, ausgeführt.

Es wurden bei $0,3595 \mathrm{Grm}$. Substanz 0,515 Grm. Kupfer gelöst, entsprechend $0,1422 \mathrm{NO}_{5}=40,71$ p.C. Salpetersäure. Hieraus folgt die Formel:

$\mathrm{CeONO}_{5}+\mathrm{NiONO}_{5}+8 \mathrm{HO}$.

$\begin{array}{lcc} & \text { Bcrechnet. } & \text { Gefunden. } \\ \mathrm{CeO} & \mathbf{1 9 , 8 9} & \mathbf{2 1 , 0 6} \\ \mathrm{NiO} & \mathbf{1 3 , 8 1} & \mathbf{1 3 , 0 2} \\ 2 \mathrm{NO}_{5} & \mathbf{3 9 , 7 8} & \mathbf{4 0 , 7 4} \\ \mathbf{8 H O} & \mathbf{2 6 , 5 1} & -\end{array}$

Salpetersaures Ceroxydul mit salpetersaurem Zinkoxyd.

Aus einer heiss gemischten Lösung von salpetersaurem Ceroxydul mit salpetersaurem Zinkoxyd erhält man beim Erkalten das Doppelsalz in schönen, grossen, völlig farblosen Krystallen. Dasselbe schliesst sich in seiner Krystallform und seinen übrigen Eigenschaften völlig den vorher beschriebenen Salzen an.

Zur Untersuchung wurden 1,005 Grm. in Wasser gelöst und mit überschüssigem Ammoniak versetzt. Das gefällte Ceroxydul ergab geglüht $0,2135 \mathrm{Ce}_{3} \mathrm{O}_{4}$, entsprechend $0,20368 \mathrm{CeO}=20,26$ p.C. Ceroxydul. In dem Filtrat mit Schwefelammonium das Zink gefällt, das Schwefelzink abfiltrirt, geglüht und in Salpetersäure gelöst, von dem ungelösten Schwefel abfiltrirt, wurde die Lösung in eine heisse Auflösung von kohlensaurem Natron eingetragen, gekocht und nach vollständiger Fällung filtrirt. Der geglühte Niederschlag ergab $0,153 \mathrm{ZnO}=15,22$ p.C. Zinkoxyd.

Zur Salpetersäurebestimmung wurden 0,452 Grm. des Doppelsalzes in einem Kolben in Wasser gelöst, dazu Salzsäure gesetzt und $\mathbf{0 , 9 2 5} \mathrm{Grm}$. Kupferblech hinzugefügt, der Kolben durch einen doppelt durchbohrten Gummipfropfen geschlossen, wovon die eine Oeffnung eine Glasröhre enthielt, die mit einem Kohlensäuregasometer in Verbindung stand; eine durch die andere Oeffnung gesteckte Glasröhre führte in einen zweiten Kolben, in welchem ebenfalls ein gewogenes Kupferblech und Salzsäure 
sich befand. Auch dieser Kolben war mit einem doppeltdurchbohrten Gummipfropfen verschlossen; aus der einen Oeffnung führte ein enges $z$ weimal gebogenes Rohr, durch die andere ging das Verbindungsrohr mit dem ersten Kolben. Als der Apparat zusammengestellt war, wurde ein lebhafter Strom Kohlensäuregases durch die Kolben getrieben, alsdann der erste Kolben vorsichtig erwärmt. Als hier die Flüssigkeit wieder. fast farblos geworden war, wurde der zweite Kolben ebenso erhitzt, alsdann die Oeffnung des engen Rohres im zweiten Kolben geschlossen und die Flamme fortgenommen.

Nachdem so der Apparat in einer Kohlensäure- $\Lambda$ tmosphäre erkaltet war, wurden die Kupferbleche herausgenommen, mit Salzsäure und nachher mit Wasser abgespült, getrocknet, gewogen, ergaben einen Gewichtsverlust von 0,614 entsprechend $0,1746 \mathrm{NO}_{5}=38,62$ p.C. Salpetersäure. Hiernach ergiebt sich die Formel:

\begin{tabular}{lcc}
\multicolumn{2}{c}{$\mathrm{CeONO}_{5}+\mathrm{ZnONO}_{5}+\mathbf{8 H O}}$. \\
Berechnet. & Gefunden. \\
$\mathrm{CeO}$ & $\mathbf{1 9 , 6 7}$ & $\mathbf{2 0 , 2 6}$ \\
$\mathrm{ZnO}$ & $\mathbf{1 4 , 8 2}$ & $\mathbf{1 5 , 2 2}$ \\
$2 \mathrm{NO}_{5}$ & $\mathbf{3 9}, 34$ & 38,62 \\
$\mathbf{8 H O}$ & $\mathbf{2 6 , 2 2}$ &
\end{tabular}

Diese salpetersauren Doppelsalze des Ceroxyduls mit den Basen der Magnesiagruppe (bis jetzt dargestellt das Magnesia-, Zink-, Mangan-, Kobalt- und Nickelsalz) zeichnen sich durch grosse Krystallisationsfähigkeit aus und zwar krystallisiren sie in regelmässig sechseckigen Taleln, doch kommen auch zuweilen hemiedrische Formen vor und häufig sind drei gegenüberliegende Seiten grösser als die drei anderen zwischen diesen liegenden.

Alle diese Salze zeigen in hohem Grade die Eigenthümlichkeit, weit über ihren Krystallisationspunkt hinaus zu erkalten, und zwar findet alsdann selbst bei heftigem Rühren mit einem glatten Glasstabe keine Ausscheidung von Krystallen Statt, sondern dieselbe erfolgte erst, sobald man einen noch so kleinen Krystall in die Lösung warf. Die Flüssigkeit erstarrt alsdann plötzlich unter be- 
trächtlicher Wärmeentwicklung zu einer festen krystallischen Masse.

Um desshalb die Salze in der zur Analyse erforderlichen Reinheit darzustellen, wurde die durch Auflösen grösserer schon ziemlich reiner Krystalle in wenig heissem Wasser erhaltene Lösung nicht zu stark eingedampft und noch vor dem völligen Erkalten ein kleiner hrystall hineingeworfen. Als darauf schnell urngerührt und bis zum völligen Erkalten das Umrühren öfter wiederholt wurde, resultirte ein feines Krystallmehl, in dem man aber mittelst der Lupe die Krystallform sehr genau erkennen konnte. Dieses Krystallmehl zwischen Fliesspapier gepresst und dann über Schwefelsäure getrocknet diente zur Analyse.

Es ist durchaus zur Erhaltung von schönen Krystallen erforderlich, dass die Lösung so neutral wie möglich sei, da man aus einer stark sauren Lösung nur äusserst schwer Krystalle erhält und diese sehr leicht zerfliessen.

Man muss daher die Flüssigkeit, aus der man gute Krystalle erhalten will, mehrmals unter Erneuerung des Wassers eindampfen, bis die Flüssigkeit nicht mehr sauer riecht; alsdann von dem ausgeschiedenen basischen Salze abfiltriren und die Lösung langsam eindampfen, wo man dann beim langsamen Erkalten schon sehr schöne, grosse Krystalle erhalten kann. Eine neutrale Lösung lässt sich sowohl mit warmem als mit kaltem Wasser verdünnen, ohne sich merklich zu trüben, während eine saure Lösung fast fortwährend durch Ausscheiden von basischen Salzen getrübt wird. Die Krystalle aus neutralen Lösungen sind ziemlich luftbeständig und lassen sich gut conserviren.

Die Analysen der salpetersauren Cerdoppelsalze bieten sehr viel Schwierigkeiten dar, einestheils durch die allgemeine Ungenauigkeit der Salpetersäurebestimmungen, anderntheils durch die Schwierigkeit, das Cer von anderen Metalloxyden zu trennen, da in die Cerniederschläge fast immer etwas von den andern Oxyden mit eingeht und durch Auswaschen nicht davon zu trennen ist. Das Wasser lässt sich aus dem Verlust beim Trocknen gar nicht 
bestimmen, da selbst nach mehrstündigem Trocknen bei $180^{\circ}$ nicht alles Wasser fortgeht und bei höherer Temperatur schon etwas Salpetersäure zersetzt wird.

\section{Ceriumplatincyanür ${ }^{*}$ ).}

Zersetzt man schwefelsaures Ceroxydul mit Baryumplatincyanür, so erhält man nach dem Eindampfen der vom schwefelsauren Baryt abfiltrirten Flüssigkeit beim Erkalten kleine glänzende, gelblich gefärbte Krystalle, die Trichroismus in Blaugrün und Gelb zeigen. Die Lösung zersetzt sich beim Eindampfen etwas und es scheidet sich Platincyanür aus. Zur Analyse wurde zur Darstellung des Salzes überschüssiges schwefelsaures Ceroxydul genommen, die Flüssigkeit im Wasserbade zur Trockne eingedampft und der Rückstand mit Alkohol ausgezogen. Das schwefelsaure Ceroxydul blieb ungelöst; die alkoholische Lösung des Salzes wurde längere Zeit mit Wasser gekocht zur Verjagung des Alkohols. Die aus der concentrirten Lösung beim Erkalten sich ausscheidenden Krystalle wiederholt zwischen Fliesspapier getracknet, da das Salz über Schwefelsäure Wasser verliert und undurchsichtig wird. Von dem so dargestellten Salze wurden 0,848 Grm. vier Stunden lang bei $150^{\circ}$ getrocknet. Hierbei ergab sich ein Gewichtsverlust von 0,165. Der Rückstand im Sauerstoffgasstrom geglüht, wog 0,5225. Dieser wurde mit Schwefelsäure digerirt und zur Trockne gebracht, nach dem Erkalten mit Wasser ausgezogen, abfiltrirt, und hinterliess 0,3225 Grn. Platin. In der Flüssigkeit wurde mit oxalsaurem Ammoniak das Ceroxydul gefällt, dieses abfiltrirt und geglüht, ergab 0,1985 Ceroxyduloxyd, entsprechend 0,16132 Cerium. Hieraus ergiebt sich die Formel: $\mathrm{CeCy}+\mathrm{PtCy}+6 \mathrm{HO}$.

$\begin{array}{lcc} & \text { Bereehnet. } & \text { Gefunden. } \\ \text { Cerium } & \mathbf{1 8 , 4} & \mathbf{1 9 , 0 2} \\ \text { Platin } & \mathbf{3 9 , 4} & \mathbf{3 8 , 0 3} \\ \text { Cyan } & \mathbf{2 0 , 6} & - \\ \text { 6HO } & \mathbf{2 1 , 6} & \mathbf{1 9 , 4 5} .\end{array}$

*) Diescs Salz ist auch von Czudnowicz dargestellt und mit gleichen Resultaten untersucht worden. (Dics. Journ. LXXX, 20.) 
Zur Bestimmung des Cyans wurde noch eine organische Verbrennung des Salzes ausgeführt. Ein $\frac{2}{3}$ Meter langes Verbrennungsrohr wurde an einem Ende zu einer dünnen Röhre ausgezogen und nach Einbringung eines Asbestpfropfens in der Länge eines halben Meters mit Kupferoxyd gefüllt. Alsdann wurde ein durch eine Schwefelsäureflasche, ein Chlorcalcium - und ein Kalirohr getrockneter und gereinigter Strom von Sauerstoffgas durch das Rohr geleitet und diess zum schwachen Rothglühen erhitzt. Nachdem auf diese Weise das Rohr, das Kupferoxyd und die darin befindliche Luft völlig getrocknet und von Kohlensäure befreit war, wurde mit dem Erhitzen aufgehört und unter Hindurchleiten von getrocknetem Sauerstoffgase erkaltete das Rohr. Alsdann wurde an dem dünn ausgezogenen Ende des Verbrennungsrohrs das gewogene Chlorcalciumrohr mittelst Kautschukverbindung befestigt, an diesem ein gewogener Geisler'scher Kaliapparat nebst Kalirohr. Am andern Ende des Rohrs, welches durch einen durchbohrten Kautschukpfropfen verschlossen war, wurde ein gewogenes Porcellanschiffchen mit gewogenem Ceriumplatincyanür eingeschoben, welches durch nochmaliges Umkrystallisiren und wiederholtes Pressen zwischen Fliespapier möglichst gereinigt und getrocknet war. Alsdann wurde das Verbrennungsrohr wieder geschlossen und die Verbindung mit dem das Sauersteffgas enthaltenden Gasometer durch die oben erwähnten Trockenapparate wieder hergestellt. Hierauf wurde das vordere Ende des Rohrs zum Glühen erhitzt, hiermit vorgeschritten bis ziemlich nahe an die Stelle, wo das Schiffchen mit Substanz lag. Als diese hierauf langsam erwärmt wurde, verwandelte sich die gelbe Farbe derselben in Weiss und kurz vor dem Erglühen wieder in Gelb. Nach dem Verglimmen war die Substanz völlig schwarz geworden und es war nicht möglich, durch starkes Glühen diese Farbe zu ändern. Als Verbrennungsofen diente der Sonnenschein'sche Apparat zur organischen Analyse.

Die Resultate der Verbrennung waren folgende:

Bei $0,459 \mathrm{Grm}$. Substanz hatte das Chlorealciumrohr 0,105 Grm., die Kaliapparate zusammen 0,1695 Grm. zugeJourn. f. prakt. Chemie. LXXXIl. 3. 
nommen. Die Substanz wog nach dem Glühen 0,293 Grm. Hieraus ergeben sich folgende Resultate nach der Formel $\mathrm{CeCy}+\mathrm{PtCy}+6 \mathrm{HO}$.

$\begin{array}{lc}\text { Berechnet. } & \text { Gefunden. } \\ \text { Wasser } 21,6 \text { p.C. } & \mathbf{2 2 , 8 7 .} \\ \text { Cyan } \quad \mathbf{2 0 , 8} " & \mathbf{2 1 , 8 2 .}\end{array}$

\section{Essigsanres Ceroxydul,}

Zu erhalten durch Aufösen von Ceroxydul, welches durch Schwefelwasserstoff reducirt ist, in Essigsäure, oder durch Zersetzung von schwefelsaurem Ceroxydul mit essigsaurem Baryt. Beim Eindampfen der filtrirten Flüssigkeit setzt sich das Salz an der Oberfläche in kleinen, dichtstrahlenförmig gruppirten Nadeln an.

Angewendet wurden 0,793 Grm. essigsauren Ceroxyduls, durch Oxalsäure das Ceroxydul gefällt, von der Flüssigkeit abfiltrirt, geglüht und gewogen, gab es 0,395 Ceroxydoxydul $=0,377$ Ceroxydul. Daraus ergiebt sich die Formel $\mathrm{CeOC}_{4} \mathrm{H}_{3} \mathrm{O}_{3}+\mathrm{HO}$.

$\begin{array}{lcc} & \text { Berechnct. } & \text { Gefunden. } \\ \text { Ceroxydul } & \mathbf{4 7 , 3 6} & \mathbf{4 7 , 5 4} . \\ \text { Essigsäure } & \mathbf{4 4 , 7 4} & \\ \text { Wasser } & \mathbf{7 , 9} & \end{array}$

Das Salz verliert das 1 Aeq. Wasser in trockner Luft (z. B. über Schwefelsäure) mit Beibehaltung seiner Krystallform.

Bei $115^{\circ} \mathrm{C}$. getrocknet, verkohlt es bei erhöhter Temperatur ohne zu schmelzen, und hinterlässt geglüht Ceroxydoxydul.

Das essigsaure Ceroxydul besitzt bei verschiedenen Temperaturen verschiedene Löslichkeit, und zwar ist es in heissem Wasser schwerer löslich als in kaltem. Untersucht wurde zuerst der Gehalt an essigsaurem Ceroxydul in einer Lösung, die acht Tage dem Verdunsten bei gewöhnlicher Temperatur ausgesetzt war und auf deren Oberfläche sich Krystalle gebildet hatten. Es wurden 10 c.C. dieser Lösung genommen, welche eine Tempera- 
tur von $27,6^{\circ} \mathrm{C}$. hatten, mit Oxalsäure gefällt, filtrirt, das oxalsaure Ceroxydul geglüht, ergab 1,316 Grm. Ceroxydoxydul, entsprechend einer Löslichkeit von 26,147 Grm. $\mathrm{CeOA}+\mathrm{HO}$ in 100 C.C. Lösung bei $27,6^{\circ} \mathrm{C}$.

Dann wurde die von den Krystallen abgegossene Flüssigkeit erhitzt, wobei sich viel Salz ausschied; es wurden 10 C.C. von dieser Lösung bei $95^{\circ} \mathrm{C}$. genommen, mit Oxalsäure gefällt, der Niederschlag abfiltrirt, geglüht, ergab 0,889 Grm. Ceroxydoxydul, diess entspricht einer Löslichkeit von $17,86 \mathrm{Grm}$. CeOĀ $+\mathrm{HO}$ in 100 c.C. der Lösung bei $95^{\circ} \mathrm{C}$.

Das Salz hat einen süssen, hernach metallischen Geschmack, dem des Bleizuckers ähnlich.

\section{Essigsaures Ceroxydoxydul.}

Versetzt man eine Lösung von schwefelsaurem Ceroxydoxydul mit essigsaurem Baryt vorsichtig so lange, als noch ein Niederschlag von schwefelsaurem Baryt entsteht, und filtrirt das Klare davon ab, so erhält man eine gelbe Flüssigkeit, in welcher Essigsäure und Ceroxydoxydul nachgewiesen werden können. Die Flüssigkeit lässt sich erhitzen und eindampfen, ohne dass das Ceroxydoxydul durch die Essigsäure reducirt wird. Bei stärkerer Concentration zersetzt sich jedoch das Ceroxydoxydul zu Ceroxydul und die Flüssigkeit wird farblos. 